Note

\title{
Advances in immunoproteomics for serological characterization of microbial antigens
}

\author{
Nandini Falisse-Poirrier ${ }^{\text {a, }}$, Virginie Ruelle ${ }^{\text {b, }}$, Benaissa ElMoualij ${ }^{\text {a }}$, Danièle Zorzi ${ }^{\text {, }}$ \\ Olivier Pierard ${ }^{\mathrm{a}}$, Ernst Heinen ${ }^{\mathrm{a}}$, Edwin De Pauw ${ }^{\mathrm{b}}$, Willy Zorzi ${ }^{\mathrm{a}, *}$ \\ ${ }^{a}$ Center of Research on Prion Proteins, Institute of Pharmacy-CHU, B36, Avenue de l'Hôpital 1, B-4000 University of Liège, Belgium \\ b Mass Spectrometry Laboratory, Sart Tilman, B6c, B-4000, University of Liège, Belgium
}

Received 2 January 2006; received in revised form 4 May 2006; accepted 15 May 2006

Available online 5 July 2006

\begin{abstract}
We propose a multi-dimensional strategy, associating immunodetection to a protein fractionating two-dimensional liquid chromatography tool, for serological characterization of microbial antigens. The originality of such immunoproteomic approaches resides in their application in large-scale studies for rapid serotyping of micro-organisms, evaluation of immunomes and could be proposed in the development and monitoring of vaccines.
\end{abstract}

(C) 2006 Elsevier B.V. All rights reserved.

Keywords: Immunoproteomics; Multi-dimension; Protein fractionation; PF2D

Immunoproteomics could be defined as the combination of any proteomic technology with an immunological data presentation. Its development is vital in an age where it is increasingly becoming urgent to identify disease biomarkers and pathogenic target antigens for the development of new drugs and vaccines. Among the current proteomic techniques available (Steel et al., 2005), 2-DE has often been chosen as the working tool in immunoproteomic applications in combination with Western-blot (Klade, 2002). In this context, two-

Abbreviations: SERPA, Serological Proteome Analysis; 2DLC, two-dimensional liquid chromatography; PF2D, Protein fractionating two-dimensional system; 2-DE, two-dimensional gel electrophoresis; $\mathrm{MM}$, apparent molecular mass; MS/MS, tandem mass spectrometry used for polypeptide micro-sequencing; $\mathrm{pI}$, isoelectric point; $2 \mathrm{D}$, two dimension

* Corresponding author. Tel.: +32 436643 27; fax: +32 43664321 .

E-mail address: Willy.Zorzi@ulg.ac.be (W. Zorzi).

${ }^{1}$ These authors have equally contributed to the article. dimensional liquid chromatography systems (2DLC) (Neverova and Van Eyk, 2005) could promote the rapid development of this area of research owing to their high potential for automation and flexibility at being interfaced with gel or liquid array-based immunological techniques and analytical systems such as tandem mass spectrometry (MS/MS) (Yan et al., 2003).

Here, we propose an immunoproteomic strategy associating an analytical protein fractionating 2DLC tool (PF2D) to immunological techniques, for the rapid characterisation of unknown antigens, immunogens and sera. The characterisation of a serum taken after a specific immunological response necessitates the establishment of its cognate antigenic repertoire. A multidimensional strategy combining PF2D with immunodetection, called PF2D-SERPA (serological proteome analysis), could hence be applied to characterize such a repertoire on the basis of $\mathrm{pI}$, hydrophobicity, antibody reactivity and relative mass. 
A variant of this strategy, i-PF2D-MS/MS, was tested to identify the antigenic target(s) of an anti-Bacillus subtilis antibody 1Bs-5 (Fig. 2A). The first step in our experimental scheme (Fig. 1A), involves the preparation of a soluble protein lysate to be injected onto the PF2D system. In our study, we used a PF2D system ('ProteomeLab PF2D', Beckman Coulter) based on the prototype of Lubman (Lubman et al., 2002). This fully automated tool employs $\mathrm{pI}$ and relative hydrophobicity as the two parameters for protein fractionation in the first and second dimension respectively. Liquid fractions obtained at the end of each dimension can be separately analyzed by immuno-blot to locate the fraction(s) that contain the antigen(s) of interest. Before performing the 2nd dimension separation, the fractions obtained after 1 st dimension can be analyzed by several techniques; in our case dot-blot and Western-blot.
Dot-blot used in first-line as a protein array provides a rapid and economical means of screening large numbers of liquid fractions for immuno-reactivity and hence reduces the number of fractions to be further analyzed. In our study, (Fig. 2B), it allowed us to narrow down our window of analysis by selecting from a total of 30 fractions, four fractions which in Western-blot resulted in only one immuno-positive fraction (Fig. 2C). This corresponded to a $\mathrm{pI}$ range of 4.72-5.02 and consisted of a single immuno-reactive protein band of interest $(\mathrm{MM} \sim 35 \mathrm{kDa})$. The higher immuno-reactivity observed in dot-blot as compared to Western-blot, could be due to differences in protein conformation brought about by variations in denaturation conditions: "partial" in dot-blot and "total" by Laemmli denaturation (Laemmli, 1970) before Western-blot. Also, the absence of protein separation in



Fig. 1. Schematic representation of immunoproteomic applications of the 'ProteomeLab PF2D' for antigenic (i-PF2D-MS/MS) and serological characterization (PF2D-SERPA) of unknown microbial antigens. (A) Antigenic characterization is realized using an antibody directed against an unknown antigen of a protein lysate. (B) Serological characterization is realized using serum of one or more individuals vaccinated (VA1 and VA2) with the same attenuated/inactivated micro-organism. 


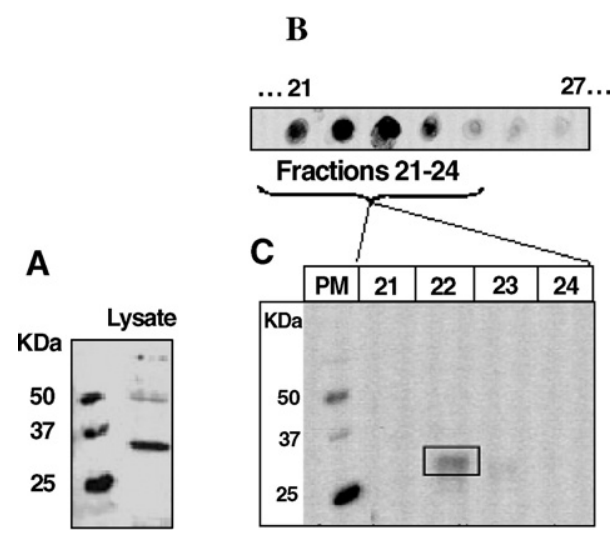

Fig. 2. (A) Western-blot of Bacillus subtilis protein lysate using 1Bs-5 serum. (B) Immuno-reactivity of a partially purified polyclonal antibody used on PF2D 1st dimension Bacillus subtilis lysate fractions, in dot-blot and (C) in Western-blot.

dot-blot as opposed to SDS-PAGE may, in some cases, result in different antibody-antigen specificity reactions. Combining both dot and Western-blot provides supplementary information regarding the immuno-reactive properties of the antibody used visà-vis the different biochemical folded states of the target antigen.

In this way, 1st dimension antigenic fractions revealed and characterized by $\mathrm{pI}$, can be selected for subsequent separation in 2 nd dimension. The resulting 2nd dimension liquid fractions are systematically examined by dot-blot and the antigenic fractions revealed are characterized by relative hydrophobicity. A Western-blot performed at this stage enhances the resolution of the $2 \mathrm{D}$ fractionation by additionally separating the antigenic fraction along the relative mass.

In our case, we applied the above procedure to the $1 \mathrm{st}$ dimension reactive fraction. By Western-blot, we identified the antigenic 2nd dimension fraction (Fig. $3 \mathrm{~A})$ and by silver nitrate-staining of the SDS-PAGE gel, used to further purify the $2 \mathrm{D}$ reactive fraction by separating the proteins along their relative mass, we isolated and excised the reactive antigen for nonambiguous peptide sequencing by LC-MS/MS (Fig. 3B). The silver-staining step after each dimension gave us a good idea of the level of purity achieved after fractionation. This step was crucial in order to increase the reliability of MS/MS sequencing. The $\mathrm{pI}$ data and relative hydrophobicity obtained from our 1 st and 2 nd dimension and the apparent molecular mass data from SDS-PAGE all contributed to the biochemical characterization of the target antigen; the latter finally identified, after isolation and sequencing of the protein band of interest by $\mathrm{MS} / \mathrm{MS}$, as flagellin (pI 4.97, nominal mass $32607 \mathrm{Da}$; SwissProt accession no. P02968).

To monitor public health, it is often necessary to characterize and identify a full set of antigens such as those targeted by an immune system (immunome) in a pathology (Klade, 2002; Madoz-Gurpide et al., 2001). In this field, techniques such as SERPA, have previously demonstrated the powerful potential of combining 2-DE with serology (Vytvytska et al., 2002). PF2D-SERPA coupled to mass spectrometry, protein preparations of pathogens can be rapidly resolved by $2 \mathrm{D}$ liquid separation and immuno-reactivity of sera, derived for example from healthy and pathogen-infected populations, can be tested by array and gel-based immunodetection. Several antigens can thus be characterized by their pI, relative hydrophobicity and relative mass, with an eventual identification by peptide sequencing, and classified on the basis of their immuno-reactivity as potential new vaccine candidates. In the PF2D-SERPA strategy (Fig. 1B), we propose a method by which the efficiency of a novel vaccine (multi-antigen targeting or preparations of attenuated or inactivated pathogen, commensal or opportunistic micro-organisms) can be evaluated by testing sera from vaccinated individuals. In Fig. 1B, vaccinated animals (VA1 and VA2), show serum reactivity different for most antigens but similar for others e.g. Fraction 3 and Fraction $\mathrm{C}$ in the diagnostic array. In our laboratory, different rabbit sera raised against $B$. subtilis, used as a model, were developed and analyzed. Firstly, a B. subtilis protein lysate was prepared, dosed and fractionated using our PF2D system. The resulting liquid fractions were then analyzed by Western-blot using sera from vaccinated individuals. This step revealed the immunogenic fractions that were then selected for separation on a silver nitrate stained SDS-PAGE gel for subsequent peptide sequencing by MS/MS. A particular serum derived after
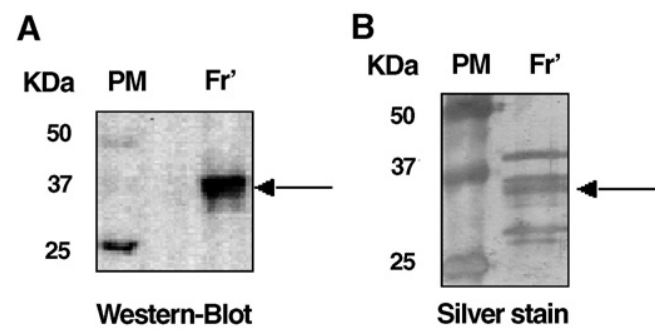

Fig. 3. (A) Western-blot and (B) Silver stain of antigenic fraction after 2nd dimension $\left(\mathbf{F r}^{\prime}\right)$. The target antigenic band was detected by Western-blot (arrow) and excised from a silver-stained gel for subsequent analysis by MS/MS. 
vaccination can hence be characterized and classified following its immunogenic pattern. Moreover, the immunogens could in turn be characterized by $\mathrm{pI}$, hydrophobicity and relative mass. Characterizing sera, derived from a number of vaccinated individuals, often reveals different serotypes that may be indicative of varying degrees of protection that a vaccine confers to a heterologous population. During the testing phases of a vaccine, it is indispensable to classify the different serotypes and their distribution in the population in order to evaluate the efficacy of a novel vaccine against pathogenic immunogens (Kyaw et al., 2000). Furthermore, comparative immunomic studies could allow the identification of commonly shared and most immunoreactive targets expressed by the pathogen agents for the development of broadly protective vaccines. This strategy could be a promising tool for the development of well-adapted vaccines to rapidly evolving pathogenic agents.

In conclusion, PF2D-SERPA and its variant iPF2D-MS/MS are immunoproteomic methods which can be proposed for multi-dimensional serological characterization of unknown microbial antigens. PF2D systems complete the existing proteomic arsenal by offering several advantageous features such as nonlaborious working procedures, versatility with regard to other analytical techniques and automation. The originality of integrating PF2D systems to immunoproteomic strategies resides in their application in large-scale studies aiming to rapidly serotype microorganisms, evaluate immunomes and could be proposed in the development and monitoring of a vaccine.

\section{Acknowledgements}

This work was supported by the 'Région Wallonne' (Contracts 14531-iPCRq, RW 981/3799 and RW 114713-iMALDI).

\section{References}

Klade, C.S., 2002. Proteomics approaches towards antigen discovery and vaccine development. Curr. Opin. Mol. Ther. 4, 216-223.

Kyaw, M.H., Clarke, S., Edwards, G.F., Jones, I.G., Campbell, H., 2000. Serotypes/groups distribution and antimicrobial resistance of invasive pneumococcal isolates: implications for vaccine strategies. Epidemiol. Infect. 125, 561-572.

Laemmli, U.K., 1970. Cleavage of structural proteins during the assembly of the head of bacteriophage T4. Nature 227, 680-685.

Lubman, D.M., Kachman, M.T., Wang, H., Gong, S., Yan, F., Hamler, R.L., O’Neil, K.A., Zhu, K., Buchanan, N.S., Barder, T.J., 2002. Two-dimensional liquid separations-mass mapping of proteins from human cancer cell lysates. J. Chromatogr., B, Anal. Technol. Biomed. Life Sci. 782, 183-196.

Madoz-Gurpide, J., Wang, H., Misek, D.E., Brichory, F., Hanash, S. M., 2001. Protein based microarrays: a tool for probing the proteome of cancer cells and tissues. Proteomics 1, 1279-1287.

Neverova, I., Van Eyk, J.E., 2005. Role of chromatographic techniques in proteomic analysis. J. Chromatogr., B, Anal. Technol. Biomed. Life Sci. 815, 51-63.

Steel, L.F., Haab, B.B., Hanash, S.M., 2005. Methods of comparative proteomic profiling for disease diagnostics. J. Chromatogr., B, Anal. Technol. Biomed. Life Sci. 815, 275-284.

Vytvytska, O., Nagy, E., Bluggel, M., Meyer, H.E., Kurzbauer, R., Huber, L.A., Klade, C.S., 2002. Identification of vaccine candidate antigens of Staphylococcus aureus by serological proteome analysis. Proteomics 2, 580-590.

Yan, F., Sreekumar, A., Laxman, B., Chinnaiyan, A.M., Lubman, D. M., Barder, T.J., 2003. Protein microarrays using liquid phase fractionation of cell lysates. Proteomics 3, 1228-1235. 